\title{
Efecto del Manejo de Identidad LGBTT+ en el Bienestar Psicológico
}

\author{
Effect of the Management of LGBTT+ Identity on Psychological Wellbeing
}

\author{
Brandon Vélez-Ríos ${ }^{1 *}$, Alfonso Martínez-Taboas ${ }^{2}$, \& Coralee Pérez-Pedrogo ${ }^{3}$ \\ 1 Universidad Albizu, San Juan, Puerto Rico. (D) https://orcid.org/0000-0001-6219-6211 \\ 2 Universidad Interamericana, Recinto Metro, Puerto Rico. (D) https://orcid.org/0000-0001-8331-0423 \\ 3 Universidad Albizu, San Juan, Puerto Rico; Universidad de Puerto Rico, Recinto de Ciencias Médicas, \\ Puerto Rico. (D) https://orcid.org/0000-0001-5502-7567 \\ * Correspondencia: velez.brandon@gmail.com.
}

Recibido: 7 marzo 2021 | Aceptado: 31 marzo 2021 | Publicado: 5 abril 2021

WWW.REVISTACARIBENADEPSICOLOGIA.COM

\section{Citar como:}

Vélez-Ríos, B., Martínez-Taboas, A., \& Pérez-Pedrogo, C. (2021). Efecto del manejo de identidad LGBTT+ en el bienestar psicológico. Revista Caribeña de Psicología, 5, e5455. https://doi.org/10.37226/rcp.v5i1.5455

\section{RESUMEN}

La presente investigación, guiada por el Modelo de Estrés de Minorías (Meyer, 2003), busca examinar el efecto del manejo de la orientación sexual o identidad de género, entiéndase divulgación u ocultación, en la relación de homonegatividad internalizada/transfobia internalizada con el bienestar psicológico en personas LGBTT+ en Puerto Rico. Se tradujeron cuatro instrumentos, dos relacionados al manejo de la identidad minoritaria (ocultación o divulgación), y dos sobre los ideales negativos internalizados, y se calculó su confiabilidad. La muestra consistió de 203 participantes, donde 165 se identificaron como cisgénero, y 38 como trans u otro género. Los hallazgos mostraron que los ideales negativos internalizados predijeron el manejo de la identidad minoritaria para las personas LGBTT+. No obstante, la relación entre los ideales negativos internalizados y el bienestar psicológico solo se confirmó para los ideales de la orientación sexual, y no de la identidad de género, lo cual sugiere que otros factores pueden explicar de forma más significativa el bienestar en las personas transgénero. Se presenta una discusión de las implicaciones de estos resultados. A su vez, se describen los retos y las limitaciones del estudio debido al COVID-19, así como recomendaciones para futuros estudios.

Palabras Claves: bienestar, bienestar psicológico, género, identidad, LGBTT+

\section{ABSTRACT}

The current study, guided by the Minority Stress Model (Meyer, 2003), seeks to examine the effect of sexual orientation or gender identity management (disclosure or concealment) in the relationship between internalized negative ideals (internalized homonegativity and internalized transnegativity) and psychological wellbeing in LGBTT+ people in Puerto Rico. Four instruments were translated from English to Spanish, two of them relating to the management of the minority identity, and two regarding the internalized negative ideals. Additionally, reliability measures were calculated for each instrument. The sample consisted of 203 participants, 165 identified as cisgendered, and 38 identified themselves as trans or another gender. Results suggested that internalized negative ideals predicted 
the minority identity management in LGBTT+ people. However, the relationship between the internalized negative ideals and psychological wellbeing was only confirmed in terms of sexual orientation and not of gender identity, which suggests that other factors may better explain wellbeing in trans people. A discussion is presented on these results' implications and the study's challenges and limitations due to the Covid-19 pandemic, and suggestions for further studies.

Keywords: gender, identity, LGBTT+, psychological wellbeing, wellbeing

\section{INTRODUCCIÓN}

Para los miembros de la comunidad Lesbiana, Gay, Bisexual, Transexual, Transgénero, Queer, Intersexuales, entre otros (LGBTT+), el proceso de salir del clóset implica el autoreconocimiento y la aceptación de la orientación sexual y/o identidad de género (APA, 2012), así como el manejo de retos asociados a la divulgación de la orientación sexual en la familia, la comunidad religiosa y otros contextos sociales (Gonsiorek, 1995). Esto cobra mayor relevancia cuando se concibe a la sociedad como un contexto heteronormativo. La heteronormatividad, según definida por Warner (1991), es "el conjunto de las relaciones de poder, por medio del cual la sexualidad se normaliza y se reglamenta en nuestra cultura, y las relaciones heterosexuales idealizadas se institucionalizan y se equiparan con lo que significa ser humano" (Lespier-Torres \& Santiago-Jhaveri, 2016, p.9). Desde esta concepción, toda aquella conducta que se desvía de las relaciones heterosexuales se puede discriminar, estigmatizar y, más aún, patologizar. Como un ejemplo de esto, el Manual Diagnóstico y Estadístico de Trastornos Mentales del 1952 hasta el 1980 clasificó la conducta homosexual como un trastorno mental (Anderson \& Holland, 2015). Aun luego de la despatologización de la homosexualidad, la ideología heteronormativa contribuye a la estigmatización de la homosexualidad y transexualidad, pues por su fuerza, se asume que todas las personas son heterosexuales y deben cumplir con los roles y expectativas que se les ha asignado (Serrato \& Balbuena, 2015).

Se ha documentado extensamente los riesgos y retos que enfrentan las personas LGBTT + , tales como la como victimización (Bariola et al., 2016; Goldblum et al., 2012), el rechazo en contextos laborales (LuiggiHernández et al., 2015), y los problemas de salud mental (Espada et al., 2012; Frisell et al., 2010; Marchueta, 2014; Martínez-Taboas et al., 2016). Muchos de estos riesgos se desenlazan en respuesta a una homonegatividad internalizada, entiéndase como la aceptación personal del estigma sexual (Pineda Roa, 2013). Meyer y Dean (1998) demostraron el efecto significativo de la homofobia internalizada en baja autoestima, depresión, ideación suicida, sentimientos de culpa, ansiedad, mayor riesgo de uso de alcohol $u$ otras sustancias y vivir en la invisibilidad. De igual manera, en Puerto Rico se han evidenciado los efectos negativos de la homonegatividad internalizada (Serrano-Nazario, 2014). Hallazgos similares se han reportado en personas transgénero, donde la internalización de esos sentimientos de autodevaluación sobre su identidad trans es perjudicial y afecta su bienestar en general (Sánchez \& Villain, 2009).

El Modelo de Estrés de Minorías (Meyer, 2003) propone que la salud mental de las personas con una identidad minoritaria se ve influenciada no tan solo por los estresores ambientales que comparten todas las personas, sino también por estresores únicos que son basados socialmente en relación a su identidad minoritaria. Por ejemplo, se ha encontrado que la religiosidad, identificada como factor de protección en diversidad de poblaciones (Koenig et al., 2012), no necesariamente aplica a la población LGBTT+. La religión ha estado relacionada con el surgimiento de conflictos entre adolescentes gays y lesbianas (Jiménez et al., 2011), y con pensamientos y comportamiento suicida (Pineda Roa, 2013).

En Puerto Rico, la religiosidad ha estado relacionada con mayores niveles de prejuicios hacia personas gays y lesbianas (Nieves-Rosa et al., 2004). Si bien la religiosidad tiene un gran impacto en el bienestar de esta población, no se deben pasar por alto otras variables contextuales como la falta de políticas públicas en pro de las personas LGBTT+, las cuales pudieran fungir como estresores únicos en relación a la identidad minoritaria. Por ejemplo, se han documentado diferencias en niveles de discriminación entre países con legislación a favor del matrimonio igualitario y aquellos países sin legislación, lo cual ha 
implicado diferencias en el empoderamiento entre integrantes de la población LGBTT+ (García et al., 2019).

En la sociedad, específicamente de Puerto Rico, las personas de la comunidad LGBTT+ frecuentemente son víctimas de marginación, prejuicios y discrímenes a causa de sus identidades sexuales y de género (Asociación de Psicología de Puerto Rico (APPR), Comité de Asuntos de la Comunidad LGBT, 2014). Estas actitudes negativas se convierten en estresores para la persona, que desde el Modelo de Estrés de Minorías (Meyer, 2003), pudieran ser parte de la explicación para la prevalencia relativamente alta de problemas de salud mental de la población LGBTT+ (Frisell et al., 2010; King et al., 2008; Lucassen et al., 2017; Marchueta, 2014; Steele et al., 2017; White et al., 2010). Ciertamente, la interacción de ese estatus minoritario con el ambiente lleva experiencias de discrimen, expectativas de rechazo, ideales negativos internalizados, y tiene serias implicaciones en el bienestar de los que las padecen.

Diversos investigadores han evaluado el bienestar psicológico. Entre ellos, se destacan los trabajos realizados por Ryff (1989) donde identifica seis dimensiones del bienestar psicológico como parte del funcionamiento del ser humano: auto-aceptación, relaciones positivas con otras personas, autonomía, dominio del entorno, propósito en la vida, y crecimiento personal. Estudios realizados en Puerto Rico han encontrado que las mujeres suelen puntuar significativamente más alto que los hombres en propósito de vida y crecimiento personal (Rosa-Rodríguez et al., 2015). Sin embargo, cuando se evaluaron estas dimensiones en personas LGBTT+ se encontró que el propósito de vida y las relaciones positivas con otros, fueron las dimensiones con puntuaciones más bajas, mientras que la de relaciones positivas se correlacionó significativamente de manera negativa con percepción de conductas discriminatorias (Luiggi-Hernández et al., 2015). No cabe duda que la exposición a estos estresores es una de las razones por las que las personas LGBTT+ a veces no divulgan su identidad sexual (Luiggi- Hernández et al., 2015).

Reconociendo la relación entre la internalización de esos ideales negativos sobre la sexualidad y esferas relacionadas al bienestar psicológico, la presente investigación, guiada por el Modelo de Estrés de Minorías (Meyer, 2003), busca examinar el efecto del manejo de la orientación sexual o identidad de género, entiéndase divulgación u ocultación, en la relación de homonegatividad internalizada/transfobia internalizada con el bienestar psicológico en personas LGBTT+ en Puerto Rico. En este estudio se propone un modelo de mediación donde los ideales negativos internalizados (homonegatividad y transfobia internalizada) predicen el bienestar psicológico de las personas LGBTT+ a través del manejo de la orientación sexual e identidad de género. En este modelo se espera que la práctica de alguna religión y estatus relacional (si actualmente se encuentran en una relación amorosa), actúen como moderadores de esta relación, ya que estas variables han sido identificadas como factores que influyen en el bienestar (Meyer \& Dean, 1998, Pineda, 2013, Sánchez \& Villain, 2009).

Si bien se ha observado un aumento en la investigación sobre personas LGBTT+ en Puerto Rico, la mayoría de estas se han enfocado principalmente en estigma, homofobia, VIH/SIDA y depresión (GonzálezRivera et al., 2019; Luiggi-Hernández et al., 2015; Martínez-Taboas et al., 2016; Rodríguez-Díaz et al., 2015; Rodríguez-Díaz et al., 2016) y poco se conoce acerca de su bienestar en general. En esta investigación se pone a prueba el Modelo de Estrés de Minorías (Meyer, 2003), donde se espera que aquellas personas que asuman su identidad sexual minoritaria presenten mejor bienestar en comparación con aquellos que la reservan u ocultan.

Según el Modelo, aquellos que opten por la divulgación contarán con mayores fuentes de apoyo y recursos, los cuales tendrán un impacto en su bienestar. Se evaluó la relación entre tener ideales negativos internalizados de su orientación sexual o identidad de género en las personas no cisgénero, el manejo de la identidad y el bienestar psicológico. Específicamente, se evaluó la divulgación de identidad minoritaria (LGBTT+) como mediador en la relación de los ideales negativos internalizados y bienestar psicológico. También se evaluó el efecto moderador de las variables práctica de alguna religión, y estatus relacional, ya que éstos se han identificado como factores que ejercen un efecto en el bienestar.

\section{Objetivos e Hipótesis de Investigación}

Los objetivos específicos del estudio son: (1) conocer si los ideales negativos internalizados se relacionan con el bienestar psicológico, (2) si el manejo de la 
identidad minoritaria media la relación entre los ideales negativos internalizados y el bienestar psicológico, (3) si la práctica de religión modera la relación entre el manejo de identidad y el bienestar, y (4) si estar en una relación amorosa modera la relación entre el manejo de identidad y el bienestar. Además, se calculó la confiabilidad de los instrumentos traducidos para el estudio. Ante este planteamiento se formulan las siguientes hipótesis:

$\mathrm{H}_{1}$ : Existe relación entre la homonegatividad internalizada/transnegatividad internalizada con el bienestar psicológico.

$\mathrm{H}_{2}$ : El manejo de la orientación sexual/identidad de género media la relación entre homonegatividad/transnegatividad internalizada y el bienestar psicológico.

$\mathrm{H}_{3}$ : La práctica de alguna religión modera la relación entre el manejo de la orientación sexual/identidad de género y el bienestar psicológico.

$\mathrm{H}_{4}$ : El estar en una relación amorosa modera la relación entre el manejo de orientación sexual/identidad de género y el bienestar psicológico.

\section{MÉTODO}

\section{Diseño de Investigación}

El diseño de esta investigación fue no-experimental, cuantitativo, transversal ya que se recogieron datos entre marzo y mayo de 2020 mediante un cuestionario en línea.

\section{Participantes}

La muestra consistió de 223 participantes. Al remover los participantes que no completaron los instrumentos, la muestra final fue de 203 voluntarios. Los criterios de inclusión fueron: ser mayor de 21 años de edad, residente en Puerto Rico por más de 2 años consecutivos y con orientación sexual de lesbiana, gay, bisexual, o pansexual, o una identidad de género transgénero, no-conforme/ queer, o no-binario (LGBTT+). Además, debían comprender y estar de acuerdo con lo expuesto en el consentimiento informado. Se excluyeron automáticamente a los participantes que no contaban con los criterios de inclusión anteriormente descritos. También fueron excluidos aquellos nacidos con genitalia ambigua.
En cuanto a identidad de género (IG), 165 (81.3\%) participantes se autoidentificaron como cisgénero, es decir, como una persona identificada con el sexo que le asignaron al nacer (APPR, 2014), 2 (1\%) como hombres trans, 9 (4.4\%) como mujeres trans, y 27 (13.3\%) participantes endosaron las opciones de transgénero/otro género. No obstante, para ver la distribución de orientación sexual (OS) pueden hacer referencia a la tabla 1.

Por otra parte, un $44.3 \%(n=90)$ endosó identificarse como agnósticos o ateos, seguido por un $23.6 \%$ ( $n=48)$ como católicos, un $13.8 \%(n=28)$ se identificó como cristianos no practicantes, y $8.9 \%(n=18)$ como protestantes. Además, un $9.4 \%(n=19)$ indicó practicar alguna otra religión como budismo, episcopal, yoruba y paganismo. Finalmente, más de la mitad $(n=$ $109 ; 53.7 \%$ ) indicaron estar en una relación amorosa al momento de participar de la investigación, mientras que un $46.3 \%(n=94)$ indicó que no.

\section{Tabla 1}

Orientación Sexual (OS) de participantes.

\begin{tabular}{lcc}
\hline & Cantidad & Porciento \\
\hline Heterosexual $^{\mathrm{a}}$ & 5 & $2.5 \%$ \\
Homosexual & 143 & $70.4 \%$ \\
Bisexual & 28 & $13.8 \%$ \\
Pansexual & 19 & $9.4 \%$ \\
Asexual & 2 & $1.0 \%$ \\
Otro & 5 & $2.5 \%$ \\
\hline
\end{tabular}

Nota. Un participante no respondió esta pregunta, por lo que la suma de participantes y porciento brinda un total de 202 participantes y un $99.6 \%$ respectivamente. ${ }^{a}=$ Dentro de la muestra de personas con una identidad transgénero, 5 personas indicaron tener una orientación sexual heterosexual.

\section{Instrumentos}

Registro de Datos Sociodemográficos. En este formulario se solicitaron nociones generales como la edad, identidad de género, orientación sexual, religión, frecuencia con la que asisten a la iglesia y si actualmente se encontraban en una relación amorosa. Basado en las respuestas marcadas, los participantes eran dirigidos a la Escala de Ocultación de Orientación Sexual o la Escala de No-Divulgación del GMSR (Medida de Estrés de Minoría de Género y Resiliencia), y su respectiva escala sobre ideales negativos internalizados. 
Manejo de Orientación Sexual. La Escala de Ocultación de Orientación Sexual es una medida compuesta de seis ítems para evaluar la evitación activa de que otros conozcan su estatus de identidad sexual minoritaria. La escala es tipo Likert puntuando desde $1=n u n c a$ a $5=$ siempre, las puntuaciones tienen un rango de 6 a 30, donde a mayor puntuación, mayor evitación activa de que otros conozcan su orientación sexual. En su desarrollo original obtuvo un índice de confiabilidad que variaba entre .77 y .79 (Jackson \& Mohr, 2016).

Homonegatividad Internalizada. La subescala de Homonegatividad Internalizada de la Escala de Identidad Lesbiana, Gay, y Bisexual (LGBIS, por sus siglas en inglés; Mohr \& Kendra, 2011) consiste de tres ítems en una escala Likert de seis puntos fluctuando entre 1 (fuertemente en desacuerdo) a 6 (fuertemente de acuerdo), para una puntuación máxima de 18, representando mayor homonegatividad internalizada. En su creación la subescala obtuvo un Alfa de Cronbach entre .86 y .87.

Identidad de Género y Transfobia Internalizada. La Medida de Estrés de Minoría de Género y Resiliencia (GMSR, por sus siglas en inglés) fue desarrollada basada en el Modelo de Estrés de Minorías (Meyer, 2003), ajustado a las experiencias de las personas transgénero y género no-conforme. La misma se compone de nueve constructos: discriminación relacionada al género, rechazo relacionado al género, victimización relacionada al género, no-afirmación de la identidad de género, transfobia internalizada, expectativas negativas de eventos futuros, no-divulgación, conectividad con la comunidad, y orgullo. De estas, se utilizaron las subescalas de transfobia internalizada y no-divulgación. La subescala de transfobia internalizada fue derivada de la subescala de vergüenza del Cuestionario de Identidad Transgénero (TGIS, por sus siglas en inglés; Bockting et al., 2014). Se compone de ocho ítems y es contestada por una escala Likert de cinco puntos que van desde fuertemente en desacuerdo a fuertemente de acuerdo, con un total máximo de 32 puntos reflejando mayor transfobia internalizada. En su creación, obtuvo un Alfa de .91. La subescala de no-divulgación contiene cinco ítems desarrollados para reflejar los medios utilizados por personas transgénero y género no-conforme de no-divulgación (Testa et al., 2014). La misma obtuvo un Alfa de .80, y utiliza una escala Likert de cinco puntos, desde totalmente en desacuerdo hasta totalmente de acuerdo, para una puntuación máxima de 25 , representando mayor evitación a divulgar la identidad de género.

Bienestar Psicológico. Se utilizó la Escala de Bienestar Psicológico de Ryff (1989), en su versión de 29 ítems en español (Díaz et al., 2006) y validada por van Dierendonck et al. (2007). La escala se compone de seis dimensiones: autoaceptación, relaciones positivas con otros, autonomía, dominio del entorno, crecimiento personal y propósito en la vida. Utilizan una escala Likert de seis puntos con puntuaciones comprendidas desde 1 (totalmente en desacuerdo) a 6 (totalmente de acuerdo). Con excepción de la dimensión dominio del entorno, todas obtuvieron un Alfa mayor de .58 en Puerto Rico (Rosa-Rodríguez et al., 2015).

Debido a que la Medida de Estrés de Minoría de Género y Resiliencia (GMSR), la Escala de Identidad Lesbiana, Gay, y Bisexual (LGBIS) y la Escala de Ocultación de Orientación Sexual (SOCS) fueron instrumentos redactados en inglés, se tradujeron al español mediante el método de Back-Translation por Brislin (1970). Este método consiste en la traducción de los instrumentos al español, y la re-traducción por otra persona al inglés, para luego evaluar la consistencia de los constructos mediante un panel de expertos en el tema. Este proceso fue llevado a cabo por el Programa Graduado de Traducción de la Universidad de Puerto Rico, recinto de Río Piedras.

\section{Procedimientos Generales}

El presente estudio fue aprobado por el Comité de Ética Institucional para la Investigación adscrito a la Universidad Albizu, San Juan, Puerto Rico. Los instrumentos fueron digitalizados a la plataforma virtual de Survey Monkey, luego de adquirir la autorización de los respectivos autores de los instrumentos previamente descritos. La plataforma facilitó un enlace directo el cual fue incluido en las promociones digitales que se difundieron a través de correos electrónicos, redes sociales y mensajería instantánea. Las promociones consistieron de una breve descripción de la investigación, los criterios de inclusión, información de contacto del investigador principal y el enlace directo a los instrumentos de autorreporte. El investigador principal envió la promoción digital mediante correos electrónicos, páginas en internet orientadas a la comunidad LGBTT+ o a la investigación, las redes 
sociales de Facebook, Twitter y WhatsApp; otras personas compartieron la promoción por los medios previamente mencionados, lo cual creó un muestreo noprobabilístico, tipo bola de nieve.

Aquellos sujetos que mostraban interés en participar, eran dirigidos al Registro de Datos Sociodemográficos. La próxima página era determinada por la respuesta que seleccionaran en la pregunta de identidad de género. Si la persona seleccionaba una opción que no fuera cisgénero, pasaba a la escala de no-divulgación y la escala de transfobia internalizada del GMSR. De marcar cisgénero, eran dirigidos a la página que contiene la SOCS y la escala de Homonegatividad Internalizada del LGBIS. Una vez los participantes completaban sus respectivas escalas, pasaban a contestar la Escala de Bienestar Psicológico (Ryff, 1989), en su versión en español de 29 ítems (Díaz et al., 2006), validada por van Dierendonck et al. (2007). Si bien la participación de este estudio no representaba riesgos mayores, al finalizar la participación aparecía información acerca de dónde solicitar servicios clínicos en caso de haber experimentado algún tipo de malestar emocional o incomodidad asociada a su participación. Participar de este estudio tomaba aproximadamente 20 minutos.

\section{Análisis de Datos}

Inicialmente, se llevaron acabo análisis descriptivos y de confiabilidad utilizando el paquete estadístico IBM SPSS versión 25 . Se examinó la confiabilidad de todos los instrumentos en la investigación, donde si recibía un valor Alfa de Cronbach de .70 o superior se considera que la escala posee un nivel de confiabilidad adecuado. Para evaluar nuestras hipótesis se hizo un modelo de mediación utilizando la herramienta de PROCESS (Hayes, 2013). Primero, el rol de mediación del manejo de la identidad minoritaria (LGBTT+), en la relación de ideales negativos internalizados y bienestar psicológico. Se utilizó un intervalo de confianza de 95\% con 5,000 muestras Bootstrap para evaluar la significancia del efecto indirecto. También se evaluó el efecto de interacción de religiosidad (si asistían o no a la iglesia) en la relación del manejo de la identidad minoritaria y bienestar psicológico. El mismo análisis se repitió para el efecto de interacción de estatus de pareja. Para examinar la dirección de alguna interacción significativa, se hubiesen graficado análisis de pendientes en diferentes valores del moderador $(+/-1 D E)$.

\section{RESULTADOS}

\section{Confiabilidad de Instrumentos}

Se calculó el Alfa de Cronbach para los instrumentos traducidos y la Escala de Bienestar Psicológico de Ryff (1989), de 29 ítems (Díaz et al. 2006, Dierendonck et al. 2007). La Escala de Ocultación de Orientación Sexual (Jackson \& Mohr, 2016), utilizada para evaluar el manejo de la orientación sexual obtuvo una confiabilidad alta $(\alpha=.81)$, lo cual superó la confiabilidad del instrumento inicial entre .77 y .79, con una correlación total por ítem corregida buena que fluctuaba entre $r=.52$ y $r=.67$. La subescala de Homonegatividad traducida (Mohr, \& Kendra, 2011), obtuvo una confiabilidad alta $(\alpha=.83)$, consistente con el Alfa de Cronbach del instrumento inicial entre .86 y .87 . La correlación total por ítem corregida que fluctuaba entre $r=.57$ y $r=.79$. Por su parte, la Escala de No-divulgación (sub-escala del GMSR; Testa et al., 2014) obtuvo una confiabilidad muy alta $(\alpha=.89)$, y correlación total por ítem corregida que fluctuaba entre .56 y .82. Esto es consistente con el Alfa obtenido en su creación de .91. La Escala de Transnegatividad Internalizada también obtuvo una confiabilidad alta $(\alpha=$ .88), con una correlación total por ítem corregida que fluctuaba entre .49 y .78. En resumen, todos los instrumentos traducidos mostraron buena confiabilidad.

En cuanto al instrumento de Ryff (1989), la Escala de Bienestar Psicológico en su versión de 29 ítems en español (Díaz et al. 2006, van Dierendonck et al. 2007) mostró tener una confiabilidad baja $(\alpha=.46)$. Al evaluar cada subescala, solo los dominios de Autoaceptación $(\alpha=.81)$ y Propósito en la Vida $(\alpha=.87)$ obtuvieron una confiabilidad alta. Los dominios de Relaciones Positivas $(\alpha=.29)$, Autonomía $(\alpha=.21)$, Dominio del Entorno ( $\alpha=-.47)$, y del Crecimiento Personal $(\alpha=-.06)$, mostraron no ser confiables y presentar inconsistencia en sus correlaciones totales por ítem. Para las estadísticas descriptivas ver tabla 2, donde en promedio las personas que contestaron la Escala de Ocultación de Orientación Sexual y la Escala de Homonegatividad Internalizada reflejaron puntuaciones bajas, lo cual indica poca ocultación y pocos ideales negativos internalizados sobre su orientación sexual. Por su parte, las personas transgénero reflejaron 
puntuaciones bajas en la escala de no-divulgación, lo cual indica que no hay una tendencia marcada en evitar la divulgación de su identidad de género, pero reflejaron puntuaciones moderadas en relación a los ideales negativos internalizados sobre su identidad de género.

Tabla 2

Estadísticas Descriptivas por prueba.

\begin{tabular}{lccc}
\hline & $\mathrm{n}$ & $\overline{\mathrm{x}}$ & $\mathrm{s}^{2}$ \\
\hline $\begin{array}{l}\text { Manejo de Orientación } \\
\text { Sexual }\end{array}$ & 164 & 9.66 & 4.388 \\
$\begin{array}{l}\text { Homonegatividad } \\
\text { Internalizada }\end{array}$ & 164 & 5.09 & 3.372 \\
$\begin{array}{l}\text { Manejo de Identidad } \\
\text { de Género }\end{array}$ & 39 & 11.74 & 5.870 \\
$\begin{array}{l}\text { Transnegatividad } \\
\begin{array}{l}\text { Internalizada } \\
\text { Bienestar Psicológico }\end{array}\end{array}$ & 39 & 17.72 & 7.847 \\
\hline
\end{tabular}

Modelo 1: Homonegatividad Internalizada y Bienestar Psicológico, con el manejo de Orientación Sexual (OS) como mediador

Se llevó a cabo un análisis de mediación utilizando PROCESS (Hayes, 2013, Modelo 4) para estimar el rol del manejo de la orientación sexual entre la homonegatividad internalizada y el bienestar psicológico. En el paso $a$ del modelo de mediación, la homonegatividad internalizada predijo significativamente el manejo de la OS, $\beta=.45, t=6.37, p<.001,95 \%$ IC [.4016, .7627]. La homonegatividad internalizada explicó el $20 \%$ de la varianza del manejo de la OS. El paso $b$ del modelo no mostró que el manejo de la orientación sexual predijo el bienestar psicológico de forma significativa, $\beta=-.11, t=-1.23, p>.05,95 \%$ IC [-.5921, .1378]. Esto quiere decir que la relación no se explica a través del manejo de la OS como se hipotetizaba. Este modelo explicó un $4 \%$ de la varianza del bienestar psicológico, por lo que la moderación con las variables de practicar religión y estatus de pareja no se pudieron evaluar en el modelo. No obstante, se incluyen los resultados del modelo de moderación en la tabla 3. En el paso $c^{\prime}$, la homonegatividad internalizada no predijo significativamente el bienestar psicológico, aun cuando el manejo de la orientación sexual está en el modelo, $\beta=.241, t=-1.54, p>.05,95 \%$ IC [-.8443, .1056]. Para paso $c$, cuando el manejo de la OS no está presente en el modelo (efecto total), la homonegatividad internalizada predijo el bienestar psicológico, $\beta=$
$-.18, t=-2.33, p=.02$. Esto confirma la primera hipótesis, y reafirma como en otras investigaciones descritas en el presente trabajo, que la homonegatividad internalizada se relaciona con el bienestar psicológico, donde a mayor homonegatividad internalizada menor bienestar psicológico. Este modelo explica el 3\% de la varianza del bienestar psicológico. El efecto indirecto de homonegatividad internalizada en el bienestar psicológico no se reflejó, $\beta=.125,95 \%$ IC [$.4114, .0927]$. Esto quiere decir que el manejo de la OS no funciona como mediador entre la homonegatividad internalizada y el bienestar psicológico. El tamaño del efecto indirecto estandarizado de $x$ en $y$ fue de $\beta=-.045,95 \%$ IC [-.145, .033].

Modelo 2: Transnegatividad Internalizada y Bienestar Psicológico, con el manejo de Identidad de Género (IG) como mediador

Se llevó a cabo un análisis de mediación utilizando PROCESS (Hayes, 2013, Modelo 4) para estimar el rol del manejo de la IG entre la transnegatividad internalizada y el bienestar psicológico. En el paso $a$ de este modelo de mediación, la transnegatividad internalizada predijo significativamente el manejo de la identidad de género, $\beta=.82, t=8.94, p<.001,95 \%$ IC $[.4783, .7627]$. La transnegatividad internalizada explicó el $68 \%$ de la varianza del manejo de la identidad de género. No obstante, el paso $b$ del modelo no mostró que el manejo de la identidad de género predijo el bienestar psicológico de forma significativa, $\beta=.051$, $t=.1738, p>.05,95 \%$ IC $[-1.0775,1.2794]$. Este modelo explicó un $2 \%$ de la varianza del bienestar psicológico, por lo que la moderación con las variables de practicar religión y estatus de pareja no se pudieron evaluar en el modelo. Los datos de la moderación se pueden observar en la tabla 3 . En el paso $c^{\prime}$, la transnegatividad internalizada no predijo significativamente el bienestar psicológico, aun cuando el manejo de la IG está en el modelo, $\beta=.435, t=-.6249, p>.05$, 95\% IC [-1.1532, .6099]. Para paso $c$, cuando el manejo de la IG no está presente en el modelo (efecto total), la transnegatividad internalizada tampoco predijo el bienestar psicológico, $\beta=-.14, t=-.8669, p>.05,95 \%$ IC $[-.6981, .2797]$. Esto rechaza la primera hipótesis, donde la transnegatividad internalizada se relacionan con el bienestar psicológico. Este modelo explica el 3\% de la varianza del bienestar psicológico. El efecto indirecto de transnegatividad internalizada en el bienestar psicológico no se reflejó, $\beta=.368,95 \%$ IC 
$[-.749, .727]$. Esto quiere decir que el manejo de la identidad de género no funciona como mediador entre la homonegatividad internalizada y el bienestar psicológico. El tamaño del efecto indirecto estandarizado de $x$ en $y$ fue de $\beta=-.253,95 \%$ IC [-.513, .499].

\section{Tabla 3}

Moderación de Práctica de Religión y Estatus de Pareja en el Bienestar Psicológico.

\begin{tabular}{|c|c|c|c|c|}
\hline \multirow[t]{2}{*}{ Interacción } & \multicolumn{2}{|c|}{$\begin{array}{l}\text { Manejo de } \\
\text { Orientación } \\
\text { Sexual }\end{array}$} & \multicolumn{2}{|c|}{$\begin{array}{l}\text { Manejo de } \\
\text { Identidad } \\
\text { de Género }\end{array}$} \\
\hline & $\beta$ & $p$ & $\beta$ & $p$ \\
\hline $\begin{array}{l}\text { Religión } \times \text { Manejo de } \\
\text { Identidad }\end{array}$ & .363 & .09 & .527 & .99 \\
\hline $\begin{array}{l}\text { Estatus de Pareja } x \\
\text { Manejo de Identidad }\end{array}$ & .332 & .358 & .478 & .114 \\
\hline
\end{tabular}

\section{DISCUSIÓN}

El propósito de este estudio era explorar el efecto del manejo de la orientación sexual e identidad de género como mediadores de la relación entre los ideales negativos internalizados (homonegatividad y transnegatividad) y el bienestar psicológico. Además, si la relación entre manejo de la identidad minoritaria (LGBTT+) y el bienestar psicológico resultaba significativa, se evaluaría el efecto moderador de practicar una religión y el efecto moderador de tener una pareja, en esta relación. Finalmente, se evaluó la confiabilidad de los instrumentos, dado que cuatro de éstos fueron traducidos al español por su idioma original ser el inglés.

Similar a lo concluido por Pineda Roa (2013), la homonegatividad internalizada ejerció un efecto negativo en el bienestar psicológico de las personas LGB, confirmando parcialmente nuestra primera hipótesis. Se encontró que la homonegatividad internalizada explicaba un 3\% del bienestar psicológico. Por otra parte, no se evidenció lo mismo con la transnegatividad internalizada, ya que esta no mostró un efecto significativo en el bienestar psicológico. Entonces, nuestra primera hipótesis que establecía los ideales negativos internalizados como relacionados al bienestar psicológico de las personas LGBTT+, solo se confirmó para los ideales internalizados de la OS y no de la IG. Estos hallazgos sugieren que otros factores pudieran explicar de forma más significativa el bienestar psicológico de las personas trans. Entre estos habría que evaluar los propuestos en investigaciones previas como la posibilidad social de transicionar (Durwood et al., 2017), historial de abuso físico y sexual (Nemoto et al., 2011), y la distinción entre el estrés de minoría sexual, resiliencia y estresores de la vida general (Testa et al., 2014). Esto cobra mayor relevancia dado que la fase de recopilación de datos ocurrió durante los meses de marzo y mayo de 2020, mientras Puerto Rico se encontraba en cuarentena por el COVID-19. Tomando esto en consideración, se cambió el lapso de tiempo que se evalúa en los instrumentos de en las pasadas dos semanas a en los pasados seis meses para intentar reflejar la realidad previa a la pandemia. No obstante, el proceso de recopilación se llevó durante el periodo de cuarentena que estuvo caracterizado por incertidumbre y ansiedad, por lo que las personas se encontraban experimentando diversos estresores de vida y esto pudo haber influenciado su atención a estresores proximales.

El manejo de la OS no explicó significativamente la varianza en el bienestar psicológico. Empero, cuando el manejo de la OS estaba en el modelo, la homonegatividad internalizada tampoco predecía significativamente el bienestar psicológico. Esto pudiera sugerir que algo del manejo de la orientación sexual influía en la relación entre homonegatividad y bienestar psicológico, por lo que una variable relacionada pudiera estar ausente del modelo, como las variables contextuales: recursos de apoyo, según el Modelo de Estrés de Minorías (Meyer, 2003), el conocimiento de la orientación sexual por parte de la familia, o los retos (aumentos de estresores) que se pueden presentar para los individuos que influyen en ese manejo de esa identidad (McConnell et al., 2018). Por lo que se rechaza nuestra segunda hipótesis donde el manejo de OS mediaría la relación, pero ofrece apoyo a la existencia de una variable relacionada que pudiera influenciar.

Los resultados identificaron que la transnegatividad internalizada explicaba un $68 \%$ de la varianza del manejo de la identidad de género, indicando que esa internalización de sentimientos negativos sobre su identidad trans afecta su manejo de la identidad. Sin embargo, ni el manejo de la identidad, ni la transnegatividad explicaron significativamente el bienestar psicológico. Además de las variables previamente mencionadas que se tendrían que evaluar, el instrumento utilizado para evaluar bienestar psicológico mostró inconsistencia en sus dominios y una pobre 
confiabilidad general. Así que, evaluar salud mental (Nemoto et al., 2011) o bienestar social (García et al., 2019) pudiera resultar en un modelo mejor ajustado a la población.

Debido a que la relación entre el manejo de la identidad minoritaria no se relacionó con el bienestar psicológico, no se pudo llevar a cabo la moderación con la práctica de alguna religión y el estatus de pareja. Es por esto que no se logró evaluar nuestra última hipótesis que proponía que ambos, individualmente, moderarían la relación. No obstante, si en el futuro se repitiera el estudio con un instrumento para evaluar bienestar psicológico con mejor confiabilidad y la inclusión de las variables previamente discutidas, sospechamos que la teoría se pudiera evaluar de forma más robusta.

\section{Limitaciones}

Varias limitaciones se desprenden del presente estudio, siendo la principal la pobre confiabilidad del instrumento utilizado para evaluar bienestar psicológico. Se recomienda un análisis factorial exploratorio para las dimensiones del bienestar psicológico y explorar las relaciones entre las variables con aquellas dimensiones que obtuvieron buena confiabilidad. Un análisis secundario con Structural Equation Modeling pudiera brindar mayor información para entender estas relaciones y rescatar si las variables evaluadas se relacionan con algunos dominios específicos como autoaceptación y propósito en la vida. De igual forma, sería beneficioso explorar el dominio de relaciones positivas con otros, dado que el Modelo de Estrés de Minoría (Meyer, 2003) propone un mayor acceso a apoyo social y recursos.

Otra limitación incluye el periodo donde se llevó a cabo la recopilación de datos pues coincidió con la cuarentena en Puerto Rico causada por la pandemia del COVID-19. Aun con la modificación de la temporalidad originalmente establecida en los instrumentos de las últimas dos semanas a los últimos seis meses (esto con la expectativa de que el participante tratara de ubicarse en un tiempo previo a la pandemia) no podemos descartar que el efecto de la cuarentena pudo haber influenciado las respuestas. No obstante, es importante resaltar que este estudio muestra grandes aportaciones entre las que se destacan la traducción de cuatro instrumentos que recibieron muy buena confiabilidad. Contar con medidas traducidas culturalmente para nuestro contexto es relevante pues informa el esfuerzo de investigaciones futuras que incluyan la evaluación de variables contextuales para un mejor modelo, así como la evaluación en la prestación de servicios.

\section{CONCLUSIÓN}

Este estudio forma parte de la literatura que apunta a la importancia de incorporar los factores contextuales en la evaluación de variables relacionadas al bienestar psicológico. Jackson y Mohr (2016) sugieren que para entender cómo el manejo de estigma interseca con los factores psicosociales, resulta relevante prestarles mayor atención a las conceptualizaciones de los constructos relacionados al manejo de estigma. El estigma, siendo generalizable a distintas situaciones y procesos, puede afectar diversas áreas relacionadas al bienestar de una persona, como el alojamiento, ganancias, y la salud (Link \& Phelan, 2001). No obstante, se debe estudiar el estigma específico que puede estar asociado a la identidad minoritaria, que no necesariamente sea equivalente a la discriminación, que dirige la atención hacia aquel que discrimina. Reafirmamos la relación entre los ideales negativos internalizados sobre la identidad LGBTT+ y el bienestar psicológico. En el caso de las personas que no se identifican como cisgénero, esa relación no se evidenció en el presente estudio. Esto no descarta la relación identificada en investigaciones previas, pero sugiere que otras variables pueden estar más relacionadas a su bienestar que los ideales, como la posibilidad social a transicionar (Durwood et al., 2017).

Financiamiento: La presente investigación no fue financiada por alguna entidad ni patrocinador.

Conflicto de Intereses: No existen conflictos de intereses de parte de los autores de la investigación.

Aprobación de la Junta Institucional para la Protección de Seres Humano en la Investigación: Universidad Albizu, San Juan, Puerto Rico.

Consentimiento o Asentimiento Informado: Todo participante completó un consentimiento informado.

Proceso de Revisión: Este estudio ha sido revisado por pares externos en modalidad de doble ciego.

\section{REFERENCIAS}

American Psychological Association. (2012). Guidelines for psychological practice with lesbian, gay, and bisexual clients. 
$\begin{array}{lll}\text { American } & \text { Psychologist, } & \text { 67(1), }\end{array}$ https://doi.org/10.1037/a0024659

Anderson, J., \& Holland, E. (2015). The legacy of medicalising “homosexuality": A discussion on the historical effects of non-heterosexual diagnostic classifications The legacy of medicalising "homosexuality": A discussion on the historical effects of nonheterosexual diag. Sensoria: A Journal of Mind, Brain \& Culture, 11(1), 4-15. https://doi.org/10.7790/sa.v11i1.405

APPR Comité de Asuntos de la Comunidad LGBT. (2014). Estándares para el trabajo e intervención en comunidades de lesbianas, gays, bisexuales e identidades trans. Asociación de Psicología de Puerto Rico.

Bariola, E., Lyons, A., \& Leonard, W. (2016). Gender-specific health implications of minority stressamong lesbians and gay men. Australian and New Zealand Journal of Public Health 40, 506-512. https://doi.org/10.1111/1753-6405.12539

Brislin, R. W. (1970). Back-translation for cross-cultural research. Journal of Cross-Cultural Psychology, 1(3), 185-216. https://doi.org/10.1177/135910457000100301

Díaz.D, Rodríguez.R, Blanco.A, Moreno.B,Gallardo.C,Valle.C y Dierendonck, D. (2006). Adaptación española de las escalas de bienestar psicológico de Riff. Psicothema, 18, 572-577. http://www.crecimientopositivo.es/Materiales/well-beingpsycothema.pdf

Durwood, L., McLaughlin, K., \& Olson, K. R. (2017). Mental health and self-worth in socially transitioned transgender youth. Journal of the American Academy of Child and Adolescent Psychiatry, 56(2), 116-123. https://doi.org/10.1016/j.jaac.2016.10.016

Espada, J. P., Morales, A., Orgilés, M., \& Ballester, R. (2012). Autoconcepto, ansiedad social y sintomatología depresiva en adolescentes españoles según su orientación sexual. Ansiedad y Estrés, 18, 31-41. https://core.ac.uk/download/pdf/61419354.pdf

Field, A. (2013). Discovering statistics using IBM SPSS Statistics (4th ed.). SAGE Publications.

Fredriksen-Goldsen, K. I., Bryan, A. E. B., Jen, S., Goldsen, J., Kim, H.-J., \& Muraco, A. (2017). The unfolding of LGBT lives: Key events associated with health and well-being in later life. The Gerontologist, 57(suppl 1), S15-S29. https://doi.org/10.1093/geront/gnw185

Frisell, T., Lichtenstein, P., Rahman, Q., \& Långström, N. (2010). Psychiatric morbidity associated with same-sex sexual behaviour: Influence of minority stress and familial factors. Psychological Medicine, 40(2), 315-324. https://doi.org/10.1017/S0033291709005996

García, C., Hombrados-Mendieta, I., Domínguez, J. M., \& Bohomoletz, P. (2019). Empoderamiento, bienestar social y discriminación del colectivo LGTB en Iberoamérica. Revista Interamericana de Psicología, 53(3), 300-312. https://journal.sipsych.org/index.php/IJP/article/download/1252/985/3628

Goldblum, P., Testa, R. J., Pflum, S., Hendricks, M. L., Bradford, J., \& Bongar, B. (2012). The relationship between gender-based victimization and suicide attempts in transgender people. Professional Psychology: Research and Practice, 43(5), 468-475. https://doi.org/10.1037/a0029605
Gonsiorek, J. C. (1995). Boundary challenges when both therapist and client are gay males. En J. C. Gonsiorek (Ed.), Breach of trust: Sexual exploitation by health care professionals and clergy. (pp.225-233). Sage.

González-Rivera, J. A., Rosario-Rodríguez, A., \& Santiago-Torres, L. E. (2019). Depresión e ideación suicida en personas de la comunidad LGBT con y sin pareja: Un estudio exploratorio. Revista Puertorriqueña de Psicología, 30(2), 254-267.

Hayes, A. F. (2013). Introduction to mediation, moderation, and conditional process analysis: A regression-based approach. The Guildford Press

Jackson, S. D., \& Mohr, J. J. (2016). Conceptualizing the Closet: Differentiating Stigma Concealment and Nondisclosure Processes. Psychology of Sexual Orientation and Gender Diversity, 3(1), 80-92. https://doi.org/10.1037/sgd0000147

Jiménez, M., Borrero, N., \& Nazario, J. (2011). Adolescentes gays y lesbianas en Puerto Rico: Procesos, efectos y estrategias. Revista Puertorriqueña de Psicología, 22, 147-173. http://www.redalyc.org/html/2332/233222354004/

King, M., Semlyen, J., Tai, S., Killaspy, H., Osborn, D., Popelyuk, D., \& Nazareth, I. (2008). A systematic review of mental disorder, suicide, and deliberate self harm in lesbian, gay and bisexual people. BMC Psychiatry, 8, 1-18. https://doi.org/10.1186/1471-244X-8-70

Koenig, H., King, D., \& Carson, V. B. (2012). Handbook of Religion and Health (2nd ed.). Oxford University Press.

Lespier-Torres, Z., \& Santiago-Jhaveri, G. (2016). Introduciendo la diversidad de nuestra comunidad. En M. Vázquez-Rivera, A. Martínez-Taboas, M. Francia-Martínez, \& J. Toro-Alfonso (Eds.), LGBT 101: Una mirada introductoria al colectivo (pp. 8-19). Publicaciones Puertorriqueñas.

Link, B. \& Phelan, J. C. (2001). Conceptualizing Stigma. Annual Review of Sociology, 27, 363-385. https://doi.org/10.1146/annurev.soc.27.1.363

Lucassen, M., Stasiak, K., Samra, R., Frampton, C. M., \& Merry, S. (2017). Sexual minority youth and depressive symptoms or depressive disorder: A systematic review and meta-analysis of population-based studies. Australian \& New Zealand Journal of Psychiatry, 51(8), 774-787. https://doi.org/10.1177/0004867417713664

Luiggi-Hernández, J. G., Laborde Torres, G. E., González Domínguez, J., Carrasquillo Sánchez, G. M., Meléndez, M. P., Castro Medina, D. M., \& González Rentas, C. D. (2015). Outing the discrimination totwars LGBT people during the hiring process: what about their well-being? Outing the discrimination towards an LGBT sample. Revista Puertorriqueña de Psicología, 26(2), 194-213.

Marchueta Pérez, A. (2014). Consecuencias del bullying homofóbico retrospectivo y los factores psicosociales en el bienestar psicológico de sujetos LGB. Revista de Investigación Educativa, RIE， 32(1), 255-271. https://dialnet.unirioja.es/servlet/articulo? codigo $=4795552 \&$ orden $=1 \&$ info $=$ link $\% 5$ Cnhttps: $/ /$ dialnet.unirioja.es/servlet/extart?codigo $=4795552$

Martínez-Taboas, A., Cruz, J., \& Padilla-Martínez, V. (2016). Salud 
mental en poblaciones LGB: ¿Cuál es el escenario y cómo podemos entenderlo? En M. Vázquez, A. Martínez-Taboas, J. Toro-Alfonso, \& M. Francia (Eds.), LGBT 101: Una mirada introductoria al colectivo. (pp. 141-161). Publicaciones Puertorriqueñas.

Martínez-Taboas, A., Jiménez-Colón, G., Torres, M., Colón, M., Méndez, J., Valdez, Y., \& González, C. (2016). LGBT y sexualidad en revistas psicológicas puertorriqueñas: Un análisis bibliométrico. Ciencias de la Conducta, 31, 73-84.

McConnell, E., Néray, B., Hogan, B., Korpak, A., Clifford, A., \& Birkett, M. (2018). "Everybody puts their whole life on facebook": Identity management and the online social networks of LGBTQ youth. International Journal of Environmental Research and Public Health, 15(6), 1078-1976. https://doi.org/10.3390/ijerph15061078

Meyer, I.H. (2003). Prejudice, social stress, and mental health in lesbian, gay, and bisexual populations: Conceptual issues and research evidence. Psychological Bulletin, 129, 674-697. https://dx.doi.org/10.1037\%2F0033-2909.129.5.674

Meyer, I.H., Dean, L. (1998). Internalized homophobia, intimacy, and sexual behavior among gay and bisexual men. En: G. M. Herek (Ed.), Stigma and sexual orientation: Understanding prejudice against lesbians, gay men, and bisexuals. (pp. 160-86). Sage.

Mohr, J. J., \& Kendra, M. S. (2011). Revision and extension of a multidimensional measure of sexual minority identity: The lesbian, gay, and bisexual identity scale. Journal of Counseling Psycho$\log y$, 58(2), 234-245. https://doi.org/10.1037/a0022858

Nemoto, T., Bödeker, B., \& Iwamoto, M. (2011). Social support, exposure to violence and transphobia, and correlates of depression among male-to-female transgender women with a history of sex work. American Journal of Public Health, 101(10), 19801988. https://doi.org/10.2105/AJPH.2010.197285

Nieves Rosa, L. (2012). Homofobia al estilo universitario. Revista Puertorriqueña de Psicología, 23, 62-76.

Pineda, C. A. (2013). Factores asociados con riesgo de suicidio de adolescentes y jovenes autoidentificados como lesbianas, gays y bisexuales: estado actual de la literatura. Revista Colombiana de Psiquiatría, 42(4), 333-349.

Rodríguez-Díaz, C. E., Jovet-Toledo, G. G., Ortiz-Sánchez, E. J., Rodríguez-Santiago, E. I., \& Vargas-Molina, R. L. (2015). Sexual health and socioeconomic-related factors among HIV-positive men who have sex with men in Puerto Rico. Archives of Sexual Behavior, 44(7), 1949-1958. https://doi-org.ucapr.cobimet3.org/10.1007/s10508-015-0481-9

Rodríguez-Díaz, C. E., Jovet-Toledo, G. G., Vélez-Vega, C. M., Ortiz-Sánchez, E. J., Santiago-Rodríguez, E. I., Vargas-Molina, R. L., Rodríguez Madera, S. L., \& Mulinelli-Rodríguez, J. J. (2016). Discrimination and health among lesbian, gay, bisexual and trans people in Puerto Rico. Puerto Rico Health Sciences Journal, 35(3), 154-159.
Rosa-Rodríguez, Y., Negrón-Cartagena, N., Maldonado-Peña, Y., Quiñones-Berrios, A., \& Toledo-Osorio, N. (2015). Dimensiones de bienestar psicológico y apoyo social percibido con relación al sexo y nivel de estudio en universitarios. Avances En Psicologia Latinoamericana, 33(1), 31-43. http://www.scielo.org.co/pdf/apl/v33n1/v33n1a03.pdf

Ryff, C. D. (1989). Beyond Ponce de Leon and life satisfaction: New directions in quest of successful ageing. International Journal of Behavioral Development, 12(1), 35-55. https://doi.org/10.1177/016502548901200102

Sánchez, F. J., \& Vilain, E. (2009). Collective self-esteem as a coping resource for male-to-female transsexuals. Journal of Counseling Psychology, 56(1), 202-209. https://doi.org/10.1037/a0014573

Serrato-Guzmán, A. N., \& Balbuena-Bello, R. (2015). Calladito y en la oscuridad. Heteronormatividad y clóset, los recursos de la biopolítica. Culturales, 3(2), 151-180.

Serrano Nazario, J.A. (2014). “Miedo a quién soy": Evaluación e intervención clínica para trabajar con la homofobia internalizada a través de la terapia afirmativa gay. En A. Martínez-Taboas y G. Bernal (Eds.), Estudio de Casos Clínicos. Contribuciones a la psicología en Puerto Rico (pp.85-107). Publicaciones Puertorriqueñas.

Steele, L. S., Daley, A., Curling, D., Gibson, M. F., Green, D. C., Williams, C. C., \& Ross, L. E. (2017). LGBT identity, untreated depression, and unmet need for mental health services by sexual minority women and trans-identified people. Journal of Women's Health, 26(2), 116-127. https://doi.org/10.1089/jwh.2015.5677

Testa, R. J., Habarth, J., Peta, J., Balsam, K., \& Bockting, W. (2014). Development of the gender minority stress and resilience measure. Psychology of Sexual Orientation and Gender Diversity, 2(1), Advance online publication. https://doi.org/10.1037/sgd0000081

Testa, R. J., Sciacca, L. M., Wang, F., Hendricks, M. L., Goldblum, P., Bradford, J., \& Bongar, B. (2012). Effects of violence on transgender people. Professional Psychology: Research and Practice, 43(5), 452-459. https://doi.org/10.1037/a0029604

Toro-Alfonso, J., \& Varas-Díaz, N. (2004). Los otros : prejuicio y distancia social hacia hombres gay y lesbianas en una muestra de estudiantes de nivel universitario. International Journal of Clinical and Health Psychology, 4, 537-551.

Warner, M. (1991). Introduction: Fear of a queer planet. Social Text, 29, 3-17. http://www.jstor.org/stable/466295

White, Y. R. G., Barnaby, L., Swaby, A., \& Sandfort, T. (2010). Mental health needs of sexual minorities in Jamaica. International Journal of Sexual Health, 22(2), 91-102. https://doi.org/10.1080/19317611003648195

Wilkinson, L., \& Pearson, J. (2009). School culture and the wellbeing of same-sex-attracted youth. Gender and Society, 23(4), 542-568. https://doi.org/10.1177/0891243209339913 